\title{
ON THE COFINALITY OF THE SMALLEST COVERING OF THE REAL LINE BY MEAGER SETS II
}

\author{
TOMEK BARTOSZYŃSKI AND HAIM JUDAH
}

(Communicated by Andreas R. Blass)

ABstract. We study the ideal of meager sets and related ideals.

\section{INTRODUCTION}

This paper continues the line of investigation started in [4] and strengthens the main result from [9].

Definition 1.1. Suppose that $\mathcal{J}$ is a $\sigma$-ideal of subsets of $2^{\omega}$. Let

$$
\operatorname{add}(\mathscr{J})=\min \{|\mathscr{A}|: \mathscr{A} \subseteq \mathscr{J} \& \bigcup \mathscr{A} \notin \mathscr{J}\}
$$

and

$$
\operatorname{cov}(\mathscr{J})=\min \left\{|\mathscr{A}|: \mathscr{A} \subseteq \mathscr{J} \& \bigcup \mathscr{A}=2^{\omega}\right\} .
$$

Let $\mathscr{M}$ and $\mathscr{N}$ denote the ideals of meager and measure zero sets respectively. The goal of this paper is to study the cofinality of the cardinal $\operatorname{cov}(\mathscr{M})$.

Recall that for a set $H \subseteq 2^{\omega} \times 2^{\omega}$ and $x, y \in 2^{\omega},(H)_{x}=\left\{y \in 2^{\omega}:\langle x, y\rangle \in\right.$ $H\}$ and $(H)^{y}=\left\{x \in 2^{\omega}:\langle x, y\rangle \in H\right\}$.

The following definition is due to Reclaw ([9]).

Definition 1.2. A set $X \subseteq 2^{\omega}$ is an $R$ set if for every Borel set $H \subseteq 2^{\omega} \times 2^{\omega}$, such that $(H)_{x} \in \mathscr{M}$ for all $x \in 2^{\omega}$,

$$
\bigcup_{x \in X}(H)_{x} \neq 2^{\omega}
$$

We will use the following representation theorem for Borel sets with meager sections:

Lemma 1.3 (Fremlin [5]). Suppose that $H \subseteq 2^{\omega} \times 2^{\omega}$ is a Borel set such that $(H)_{x}$ is meager for all $x$. Then there exists a sequence of Borel sets $\left\{G_{n}: n \in\right.$ $\omega\} \subseteq 2^{\omega} \times 2^{\omega}$ such that

Received by the editors September 18, 1993 and, in revised form, September 24, 1993.

1991 Mathematics Subject Classification. Primary 04A20.

Key words and phrases. Meager sets, cardinal invariants.

Research partially supported by the Israel Academy of Science, Basic Research Foundation. 
(1) $\left(G_{n}\right)_{x}$ is a closed nowhere dense set for all $x \in 2^{\omega}$,

(2) $H \subseteq \bigcup_{n \in \omega} G_{n}$.

Proof. For completeness we present a sketch of the proof here.

Let $\mathscr{G}$ be the family of Borel subsets $G$ of $2^{\omega} \times 2^{\omega}$ such that $(G)_{x}$ is open for every $x \in 2^{\omega}$.

Let $\mathcal{J}$ be the $\sigma$-ideal of subsets of the plane generated by Borel sets $F$ such that $(F)_{x}$ is closed nowhere dense for all $x$.

Consider the family $\Sigma$ of subsets $E$ of the plane such that there are two Borel sets $G, H$ of which $G \in \mathscr{G}, H \in \mathcal{J}$, and $E \triangle G \subseteq H$. Clearly $\Sigma$ contains all open sets and is closed under countable unions. We want to show that $\Sigma$ is also closed under complements.

For a set $G \in \mathscr{G}$ let

$$
G^{\prime}=\left\{\langle x, y\rangle: y \text { is an interior point of } 2^{\omega} \backslash(G)_{x}\right\} .
$$

Note that $\left(2^{\omega} \times 2^{\omega}\right) \backslash\left(G \cup G^{\prime}\right)$ is a set whose vertical sections are closed and nowhere dense. It follows that in order to show that $\Sigma$ is closed under complements it is enough to check that $G^{\prime}$ is a Borel set.

Let $\left\{U_{n}: n \in \omega\right\}$ be a recursive enumeration of a countable base for the family of open subsets of $2^{\omega}$.

Note that the following are equivalent:

(1) $\langle x, y\rangle \in G^{\prime}$,

(2) $\exists n\left(y \in U_{n} \& \forall z\left(z \notin U_{n} \vee\langle x, z\rangle \notin G\right)\right)\left(\Pi_{1}^{1}\right)$,

(3) $\exists n\left(y \in U_{n} \& \forall m\left(U_{n} \cap U_{m}=\emptyset \vee \exists z\left(z \in U_{m}\langle x, z\rangle \notin G\right)\right)\right)\left(\Sigma_{1}^{1}\right)$.

That shows that $G^{\prime}$ has a $\Delta_{1}^{1}$ definition which means that it is a Borel set.

So $\Sigma$ is a $\sigma$-algebra which contains every Borel set. Now if $E$ is a Borel set with meager vertical sections, then associated $G$ must be empty. In particular, $E \in \mathcal{J}$.

\section{2. $R$ SETS AND THE COFINALITY OF $\operatorname{cov}(\mathscr{M})$}

In this section we will study $R$ sets and show that $R$ sets form an ideal closely related to the ideal of meager sets.

Definition 2.1. A set $X \subseteq 2^{\omega}$ has Rothberger's property (is a $C^{\prime \prime}$ set) if for every sequence of open covers of $X,\left\{\mathscr{G}_{n}: n \in \omega\right\}$, there exists a sequence $\left\{U_{n}: n \in \omega\right\}$ with $U_{n} \in \mathscr{I}_{n}$ such that $X \subseteq \bigcup_{n \in \omega} U_{n}$.

Rothberger's property is the topological version of strong measure zero. We have the following:

Theorem 2.2 (Fremlin, Miller [7]). The following are equivalent:

(1) $X \subseteq 2^{\omega}$ has Rothberger's property,

(2) $X$ has strong measure zero with respect to every metric which gives $X$ the same topology.

Let $C^{\prime \prime}$ be the collection of subsets of $2^{\omega}$ which have Rothberger's property. It is easy to see that $C^{\prime \prime}$ is a $\sigma$-ideal. 
Theorem 2.3. The following conditions are equivalent:

(1) $X$ is an $R$ set,

(2) for every Borel function $x \rightsquigarrow f^{x} \in \omega^{\omega}$ there exists a function $g \in \omega^{\omega}$ such that

$$
\forall x \in X \exists^{\infty} n f^{x}(n)=g(n) .
$$

(3) Every Borel image of $X$ has Rothberger's property.

Proof. (1) $\rightarrow(2)$ Suppose that $x \leadsto f^{x} \in \omega^{\omega}$ is a Borel mapping. Let $H=$ $\left\{\langle x, h\rangle \in X \times \omega^{\omega}: \forall \forall^{\infty} n h(n) \neq f^{x}(n)\right\}$. Clearly $H$ is a Borel set with all $(H)_{x}$ meager, and if $g \notin \bigcup_{x \in X}(H)_{x}$, then $g$ has required properties.

$(2) \rightarrow(3)$ Suppose that $Y$ is a Borel image of $X$. Let $\left\{\mathscr{G}_{n}: n \in \omega\right\}$ be a sequence of open covers of $Y$. Suppose that $\mathscr{G}_{n}=\left\{G_{n}^{m}: m \in \omega\right\}$ for $n \in \omega$. For $y \in Y$ let $f^{y} \in \omega^{\omega}$ be the function defined as

$$
f^{y}(n)=\min \left\{m: y \in G_{n}^{m}\right\} \text { for } n \in \omega .
$$

Mapping $y \leadsto f^{y}$ is Borel. Thus there exists a function $f \in \omega^{\omega}$ such that

$$
\forall y \in Y \exists^{\infty} n f^{y}(n)=f(n) .
$$

Clearly the sequence $\left\{G_{n}^{f(n)}: n \in \omega\right\}$ has required properties.

$(3) \rightarrow(2)$ Suppose that $x \leadsto f^{x} \in \omega^{\omega}$ is a Borel mapping. Clearly $Y=$ $\left\{f^{x}: x \in X\right\}$ has Rothberger's property in $\omega^{\omega}$. Consider the families

$$
\mathscr{G}_{n}=\left\{G_{n}^{m}: m \in \omega\right\} \text { where } G_{n}^{m}=\left\{h \in \omega^{\omega}: h(n)=m\right\} .
$$

The selector chosen for this sequence of coverings immediately gives us the function which meets every $f^{x}$ in at least one point.

To get the function we are looking for we have to split $\omega$ into infinitely many pieces and apply the above construction to each one of them.

(2) $\rightarrow$ (1) We will need several lemmas. To avoid repetitions let us define:

Definition 2.4. Suppose that $X \subseteq 2^{\omega}$. $X$ is nice if for every Borel function $x \leadsto f^{x} \in \omega^{\omega}$ there exists a function $g \in \omega^{\omega}$ such that

$$
\forall x \in X \exists^{\infty} n f^{x}(n)=g(n) .
$$

We will show first that:

Lemma 2.5. Suppose that $X$ is nice. Then for every Borel function $x \leadsto\left\langle Y^{x}, f^{x}\right\rangle$ $\in[\omega]^{\omega} \times \omega^{\omega}$ there exists $g \in \omega^{\omega}$ such that

$$
\forall x \in X \exists^{\infty} n \in Y^{x} f^{x}(n)=g(n) .
$$

Proof. Suppose that a Borel mapping $x \rightsquigarrow\left\langle Y^{x}, f^{x}\right\rangle$ is given. Let $y_{n}^{x}$ denote the $n$-th element of $Y^{x}$ for $x \in X$. For every $x \in X$ define a function $h^{x}$ as follows:

$$
h^{x}(n)=f^{x} \mid\left\{y_{0}^{x}, y_{1}^{x}, \ldots, y_{n}^{x}\right\} \text { for } n \in \omega .
$$

Since the mapping $x \leadsto h^{x}$ is Borel and functions $h^{x}$ can be coded as elements of $\omega^{\omega}$, there is a function $h$ such that

$$
\forall x \in X \exists^{\infty} n h^{x}(n)=h(n) .
$$

Without loss of generality we can assume that $h(n)$ is a function from an $(n+1)$-element subset of $\omega$ into $\omega$. 
Define $g \in \omega^{\omega}$ in the following way. Choose inductively

$$
z_{n} \in \operatorname{dom}(h(n)) \backslash\left\{z_{0}, z_{1}, \ldots, z_{n-1}\right\} \text { for } n \in \omega .
$$

Let $g$ be any function such that $g\left(z_{n}\right)=h(n)\left(z_{n}\right)$ for $n \in \omega$.

We show that the function $g$ has the required properties. Suppose that $x \in X$. Notice that the equality $h^{x}(n)=h(n)$ implies that

$$
f^{x}\left(z_{n}\right)=g\left(z_{n}\right) \text { and } z_{n} \in Y^{x} .
$$

That finishes the proof since $h^{x}(n)=h(n)$ for infinitely many $n \in \omega$.

Lemma 2.6. Suppose that $X$ is nice. Then for every Borel mapping $x \rightsquigarrow f^{x} \in$ $\omega^{\omega}$ there exists an increasing sequence $\left\{n_{k}: k \in \omega\right\}$ such that

$$
\forall x \in X \exists^{\infty} k f^{x}\left(n_{k}\right)<n_{k+1} .
$$

Proof. Suppose that the lemma is not true and let $x \leadsto f^{x}$ be the witness. Without loss of generality we can assume that $f^{x}$ is increasing for all $x \in X$. To get a contradiction we will define a Borel mapping $x \leadsto g^{x} \in \omega^{\omega}$ such that $\left\{g^{x}: x \in X\right\}$ is a dominating family. That will contradict the assumption that $X$ is nice.

Define

$$
g^{x}=\max \{\underbrace{f^{x} \circ f^{x} \circ \cdots \circ f^{x}}_{j \text { times }}(i): i, j \leq n\} \text { for } n \in \omega .
$$

Suppose that $g \in \omega^{\omega}$ is an increasing function. By the assumption there exist $x \in X$ and $k_{0}$ such that

$$
\forall k \geq k_{0} f^{x}(g(k)) \geq g(k+1) .
$$

In particular,

$$
\forall k \geq g\left(k_{0}\right) g(k) \leq g^{x}(k)
$$

which finishes the proof.

Lemma 2.7. Suppose that $X$ is nice. Then for every Borel mapping $x \leadsto Y^{x} \in$ $[\omega]^{\omega}$ there exists a set $Y=\left\{u_{n}: n \in \omega\right\}$ such that

(1) $u_{n+1} \geq u_{n}+2$ for all $n$,

(2) $\forall x \in X\left|Y \cap Y^{x}\right|=\aleph_{0}$.

Proof. By applying Lemma 2.6, we can find an increasing function $f \in \omega^{\omega}$ such that

$$
\forall x \in X\left|Y^{x} \backslash\{f(n): n \in \omega\}\right|=\aleph_{0} .
$$

Let $A_{0}=\{2 k: k \in \omega\}$ and $A_{1}=\{2 k+1: k \in \omega\}$. Define Borel mapping $x \leadsto\left\langle Z^{x}, g^{x}\right\rangle \in[\omega]^{\omega} \times 2^{\omega}$ as follows: $Z^{x}=\operatorname{dom}\left(g^{x}\right)$ and for $n \in \omega$,

$$
g^{x}(n)= \begin{cases}0 & \text { if } Y^{x} \cap(f(n), f(n+1)) \cap A_{0} \neq \emptyset, \\ 1 & \text { if } Y^{x} \cap(f(n), f(n+1)) \cap A_{1} \neq \emptyset, \\ & \text { undefined otherwise. }\end{cases}
$$

Note that the first two conditions of this definition are not exclusive. We use either value when that happens. 
By Lemma 2.7, there exists a function $h \in 2^{\omega}$ such that

$$
\forall x \in X \exists^{\infty} n \in Z^{x} h(n)=g^{x}(n) .
$$

Define

$$
Y=\bigcup_{n \in \omega}\left((f(n), f(n+1)) \cap A_{h(n)} .\right.
$$

It is clear that $Y$ has required properties.

We now return to the proof that (2) implies (1). Let $H \subseteq 2^{\omega} \times 2^{\omega}$ be a Borel set with all fibers $(H)_{x}$ meager. Using 1.3, find Borel sets $\left\{F^{n}: n \in \omega\right\}$ such that $H \subseteq \bigcup_{n \in \omega} F^{n}$ and $\left(F^{n}\right)_{x}$ is closed nowhere dense for $x \in 2^{\omega}$, $n \in \omega$.

For $x \in X$, define

$$
s_{n}^{x}=\min \left\{s \in 2^{<\omega}: \forall t \in 2^{<n} \forall j \leq n\left[t^{\frown} s\right] \cap\left(F^{j}\right)_{x}=\emptyset\right\} \text { for } n \in \omega
$$

(where the minimum is taken with respect to some enumeration of $2^{<\omega}$ ).

By Lemma 2.6, there exists a sequence $\left\{n_{k}: k \in \omega\right\}$ such that

(1) $n_{k+1}>\sum_{i=0}^{k} n_{i}$, for all $k$,

(2) $\forall x \in X \exists^{\infty} n\left|s_{n_{k}}^{x}\right|<n_{k+1}$.

For $x \in X$ let $Z^{x}=\left\{k:\left|s_{n_{k}}^{x}\right|<n_{k+1}\right\}$. By Lemma 2.5, there exists a sequence $\left\langle s_{k}: k \in \omega\right\rangle$ such that

$$
\forall x \in X \exists^{\infty} k \in Z^{x} s_{n_{k}}^{x}=s_{k} .
$$

Without loss of generality we can assume that $\left|s_{k}\right|<n_{k+1}$ for all $k$. Define mapping $x \leadsto Y^{x}$ by

$$
Y^{x}=\left\{k \in Z^{x}: s_{k}=s_{n_{k}}^{x}\right\} .
$$

Let $Y$ be a set obtained by applying Lemma 2.7 to this family. Define

$$
z=s_{l_{0}} \frown s_{l_{1}} \frown s_{l_{2}} \frown \ldots,
$$

where $l_{0}<l_{1}<l_{2}<\cdots$ is the increasing enumeration of $Y$. Note that if $l_{k+1} \in Y \cap Y^{x}$, then

$$
\left|s_{l_{0}} \frown s_{l_{1}} \frown \ldots \frown s_{l_{k}}\right|<\sum_{j \leq l_{k}} n_{l_{j}+1}<n_{l_{k}+2} \leq n_{l_{k+1}}
$$

and

$$
s_{l_{k+1}}=s_{n_{k+1}}^{x} .
$$

In other words, $\left[s_{l_{0}} \frown s_{l_{1}} \frown \ldots s_{l_{k+1}}\right] \cap\left(F^{i}\right)_{x}=\emptyset$ for $i \leq l_{k+1}$. But that clearly implies that $z \notin \bigcup_{n \in \omega}\left(F^{n}\right)_{x}$, which finishes the proof.

As a corollary we get the following:

Theorem 2.8 (Reclaw [9]). Every Luzin set is an $R$ set.

Proof. It is well known that every Borel image of a Luzin set has Rothberger's property.

Theorem 2.9. $\operatorname{cf}(\operatorname{cov}(\mathscr{M})) \geq \operatorname{add}(R) \geq \operatorname{add}\left(C^{\prime \prime}\right) \geq \operatorname{add}(\mathscr{N})$.

Proof. We will start with the following lemma. 
Lemma 2.10. There exists a Borel set $H \subseteq 2^{\omega} \times 2^{\omega}$ with such that all $(H)_{x}$ are meager and for every set $A \in \mathscr{M}$ there exists $x \in 2^{\omega}$ such that $A \subseteq(H)_{x}$.

Proof. For $z \in 2^{\omega}$ and $f \in \omega^{\omega}$ define a set

$$
\begin{aligned}
B(z, f)=\left\{t \in 2^{\omega}: \forall^{\infty} n \exists j \in[\widetilde{f}(n), \tilde{f}(n+1)) t(j)\right. & \neq z(j)\}, \\
& \text { where } \widetilde{f}(n)=\sum_{k=0}^{n} f(k) .
\end{aligned}
$$

It is well known (see [2]) that the family $\left\{B(z, f): z \in 2^{\omega}, f \in \omega^{\omega}\right\}$ is a basis of $\mathscr{M}$.

Fix a Borel surjection $t \leadsto\left\langle z_{t}, f_{t}\right\rangle \in 2^{\omega} \times \omega^{\omega}$ and let $H$ be a set such that $(H)_{t}=B\left(z_{t}, f_{t}\right)$ for all $t \in 2^{\omega}$.

Suppose that $\mathscr{A} \subseteq \mathscr{M}$ is a family of meager sets of $\operatorname{size} \operatorname{cov}(\mathscr{M})$ which covers $2^{\omega}$. For $F \in \mathscr{A}$ let $x_{F} \in 2^{\omega}$ be such that $F \subseteq(H)_{x_{F}}$. Suppose that $\operatorname{cf}(\operatorname{cov}(\mathscr{M}))=\kappa$. It follows that $X=\left\{x_{F}: F \in \mathscr{A}\right\}$ is the union of $\kappa$ many sets $X_{\alpha}$ of size smaller than $\operatorname{cov}(\mathscr{M})$. Clearly each set $X_{\alpha} \in R$ and $X \notin R$. Thus $\kappa \geq \operatorname{add}(R)$ which proves the first inequality.

The second inequality follows immediately from Theorem 2.3.

The third inequality is due to Carlson. We will prove it here for completeness. We will use the following fact (see [2] or [1]):

Theorem 2.11. $\operatorname{add}(\mathcal{N})$ is the smallest size of the family $F \subseteq \omega^{\omega}$ such that there is no function $S: \omega \longrightarrow[\omega]^{<\omega}$ with $|S(n)| \leq n$ for all $n$, such that

$$
\forall f \in F \forall^{\infty} n f(n) \in S(n) \text {. }
$$

Suppose that $\left\{X_{\alpha}: \alpha<\kappa<\operatorname{add}(\mathcal{N})\right\}$ is a family of $C^{\prime \prime}$ sets. We will show that $X=\bigcup_{\alpha<\kappa} X_{\alpha}$ is a $C^{\prime \prime}$ set.

Let $\left\{\mathscr{G}_{n}: n \in \omega\right\}$ be a sequence of open coverings of $X$. Assume that $\mathscr{G}_{n}=\left\{U_{m}^{n}: m \in \omega\right\}$ for $n \in \omega$. Let $r(n)=1+2+\cdots+(n-1)$. Define for $n \in \omega$ the family

$$
\widetilde{\mathscr{G}}_{n}=\left\{\widetilde{U}_{s}^{n}: s \in \omega^{[r(n), r(n+1))}\right\}, \text { where } \widetilde{U}_{s}^{n}=\bigcap_{j=r(n)}^{r(n+1)} U_{s(j)}^{j} .
$$

Note that $\tilde{\mathscr{G}}_{n}$ is also a cover of $X$. By the assumption, for every $\alpha<\kappa$ there exists a function $f_{\alpha}$ such that $\left\{\widetilde{U}_{f_{\alpha}(n)}^{n}: n \in \omega\right\}$ is a covering of $X_{\alpha}$.

Since $\kappa<\operatorname{add}(\mathcal{N})$, by Theorem 2.11, there exists a function $S: \omega \longrightarrow \omega^{<\omega}$ such that

$$
\forall \alpha<\kappa \forall^{\infty} n f_{\alpha}(n) \in S(n) .
$$

Without loss of generality we can assume that $S(n)$ consists of at most $n$ sequences of length $n$. Let $f$ be a function which agrees at least once on the $n$-element interval $[r(n), r(n+1))$ with each of the $n$ functions in $S(n)$. Clearly $\left\{U_{f(n)}^{n}: n \in \omega\right\}$ is the covering of $X$ we were looking for.

Remarks and questions. (1) Is $\operatorname{cf}(\operatorname{cov}(\mathscr{M})) \geq \operatorname{add}(\mathscr{M})$ ? In other words, is $\operatorname{cf}(\operatorname{cov}(\mathscr{M})) \geq \mathfrak{b}$ ? 
(2) The first inequality in Theorem 2.9 also holds when we replace category by measure. Unfortunately we do not know if $R$ sets defined for measure form an ideal. (For more see [8] and [3].)

(3) It is consistent that $\operatorname{add}(\mathscr{M})>\operatorname{add}\left(C^{\prime \prime}\right)([6])$.

(4) Is it consistent that $\operatorname{add}\left(C^{\prime \prime}\right)>\operatorname{add}(\mathscr{N})$ ? $\operatorname{add}(R)>\operatorname{add}(\mathscr{N})$ ?

\section{REFERENCES}

1. Tomek Bartoszyński, Additivity of measure implies additivity of category, Trans. Amer. Math. Soc. 281 (1984), 209-213.

2. Tomek Bartoszyński and Haim Judah, Set theory: On the structure of the real line, Ark. Peters (to appear).

3. _ Borel images of sets of reals, Real Anal. Exchange (submitted).

4. _ On the cofinality of the smallest covering of the real line by meager sets, J. Symbolic Logic 54 (1989), 828-832.

5. David H. Fremlin, The partial orderings in measure theory and Tukey ordering, Note Mat. (to appear).

6. Haim Judah and Saharon Shelah, MA( $\sigma$-centered): Cohen reals, strong measure zero sets and strongly meager sets, Israel J. Math. 68 (1989), 1-17.

7. Arnold W. Miller and David H. Fremlin, On some properties of Hurewicz, Menger, and Rothberger, Fund. Math. 129 (1988), 17-33.

8. Janusz Pawlikowski, All Sierpinski sets are strongly meager, Arch. Math. Logic (to appear).

9. Ireneusz Reclaw, Every Lusin set is undetermined in point-open game, Fund. Math. 144 (1994), 43-54.

Department of Mathematics, Boise State University, Boise, Idaho 83725

E-mail address: tomek@math.idbsu.edu

Abraham Fraenkel Group for Mathematical Logic, Department of Mathematics, Bar ILAN UNIVERSITY, 52900 RAMAT GAN, ISRAEL

E-mail address: judah@bimacs.cs.biu.ac.il 